\title{
Numerical Study of Interface Tracking for the Unsteady Flow of Two Immiscible Micropolar and Newtonian Fluids Through a Horizontal Channel with an Unstable Interface
}

\author{
Rajesh Kumar Chandrawat ${ }^{1, *}$, Varun Joshi ${ }^{1}$, and O. Anwar Bég ${ }^{2}$ \\ ${ }^{1}$ Department of Mathematics, Lovely Professional University Jalandhar, 144411, India \\ ${ }^{2}$ Professor and Director Metaphysical Engineering Sciences Group (MPESG), Department of MechanicallAeronautical \\ Engineering, School of Science, Engineering, Environment (SEE), Salford University, Manchester, M54WT, UK
}

The dynamics of the interaction between immiscible fluids is relevant to numerous complex flows in nature and industry, including lubrication and coating processes, oil extraction, physicochemical separation techniques, etc. One of the most essential components of immiscible flow is the fluid interface, which must be consistently monitored. In this article, the unsteady flow of two immiscible fluids i.e., an Eringen micropolar and Newtonian liquid is considered in a horizontal channel. Despite the no-slip and hyper-stick shear stress condition at the channel edge, it is accepted that the liquid interface is dynamic, migrating from one position to the next and possibly get absolute change; as a result, The CS (continuum surface) model is integrated with the single moment equation based on the VOF (volume of fluid) approach to trace the interface. The immiscible fluids are considered to flow under three applied pressure gradients (constant, decaying, and periodic) and flow is analyzed under seamless shear stress over the entire interface. The modified cubic b-spline differential quadrature method (MCB-DQM) is used to solve the modeled coupled partial differential equations for the fluid interface evolution. The advection and tracking of the interface with time, wave number, and amplitude are illustrated through graphs. It is observed that the presence of micropolar parameters affects the interface with time. The novelty of the current study is that previous studies (which considered the smooth and unstable movement of the micropolar fluid, the steady stream of two immiscible fluids, and interface monitoring through different modes) are extended and generalized to consider the time-dependent flow of two immiscible fluids namely Eringen micropolar and Newtonian with a moving interface in a horizontal channel. For the decaying pressure gradient case, which requires more time to achieve the steady-state, the peak of the waves resembles those for the constant pressure gradient case. The interface becomes steady for a more extensive time when a constant pressure gradient is applied. The interface becomes stable quickly with time as the micropolar parameter is decreased for the constant pressure gradient case i.e., weaker micropolar fluids encourage faster stabilization of the interface. With periodic pressure gradient, the interface takes more time to stabilize, and the crest of the waves is significantly higher in amplitude compared to the constant and decaying pressure cases. The simulations demonstrate the excellent ability of MCB-DQM to analyze complex interfacial immiscible flows.

KEYWORDS: Immiscible Fluids, Unsteady Flow, Interface Tracking, Volume-of-Fluid Method, Modified Cubic B-Spline, Differential Quadrature Method, Micropolar, Newtonian.

\section{INTRODUCTION}

Many commercial and technological fluids do not function as Newtonian fluids- they exhibit non-Newtonian characteristics. Various mathematical models have therefore been

*Author to whom correspondence should be addressed

Email: rajesh.16786@1pu.co.in

Received: 21 May 2021

Accepted: 1 August 2021 developed to describe non-Newtonian fluids which include viscoelastic, and polar (couple stress) fluids. An alternative and more elegant formulation are the micropolar fluid model which has stimulated considerable interest in rheological modeling worldwide. Eringen pioneered the theory of micropolar fluids in the 1960s. ${ }^{1}$ A remarkable aspect of the Eringen model is the competency to depict the fluid microstructure, including stresses which are neglected 
in the Newtonian (Navier-Stokes classical viscous flow) model and most rheological models. The microstructure is simulated via the microrotation vector, which is extraneous to the vorticity vector that creates unsymmetric stress. Microelements of the micropolar fluid can therefore sustain gyratory motions i.e., spin. Micropolar fluid dynamics has therefore been effectively used in various applications, including hemodynamics, nuclear reactor slurry flows, liquid crystals, lubricants, gels, greases, etc. Peddieson ${ }^{2}$ explored the micropolar model for turbulent channel flow analysis. Thermal coating flows with micropolar fluids in the presence of cross-diffusion effects were studied numerically by Bég et al. ${ }^{3}$ Micropolar blood flows were examined comprehensively by Kang and Eringen. ${ }^{4}$ Devakar and Iyerger ${ }^{5}$ studied the time-dependent flow of micropolar liquid in channels. Mekheimer and $\operatorname{Kot}^{6}$ analyzed the stenotic hemodynamic flow of micropolar fluids with applications in diseased tapered arteries. Srinivasa and Shiferaw ${ }^{7}$ used the Homotopic analysis method to compute the effects of cross-diffusion on micropolar convection flow in a channel. Wang et al. ${ }^{8}$ examined the motion of micropolar fluids into a micro parallel system with applications in industrial flow separation in chemical engineering systems.

Apart from the micro-structured element, the efficiency of energy transfer in the fluid has been strengthened by the suspension of the nanoparticle. Using the magnetic field and nanofluid, Selimefendigil et al. ${ }^{9}$ improved the thermal efficiency of the separated flow. The flow behavior of hybrid nanofluids was examined by Dogonchi et al. ${ }^{10}$ in an embedded system comprising magnetic field effects. The problem of heat exchange among fluid and hot surfaces within a smooth air channel was addressed by Menni et al. ${ }^{11}$ Raza et al. ${ }^{12}$ investigated the cumulative influence of heat radiation and a magneto force on molybdenum disulfide nanofluid in a conduit with shifting boundaries. The influence of Hall and ion slip on the MHD flow of nanofluids through the permeable rotating plate with a steady heat source were investigated by Krishna et al. ${ }^{13}$ Hydrodynamic and thermal characterization of turbulent forced flows of pure water, pure ethylene glycol, and liquid glycol mixtures diffused by $\mathrm{Al}_{2} \mathrm{O}_{3}$ microparticles were performed by Menni et al. ${ }^{14}$ Shashikumar et al. ${ }^{15}$ discovered the influence of various alloy nanocrystals on magnetic materials' microchannel flow. Kumar et al. ${ }^{16}$ examined The time-independent flow of an incompressible, optically thin fluid over a vertical plate was using MHD convection flow. Menni et al. ${ }^{17}$ described the findings of a computational investigation of forced-convection thermal expansion in heat exchanger ducts with various forms. Chamkha et al. ${ }^{18}$ performed the simulation of unsteady conjugated natural convection of hybrid liquid emulsion of $\mathrm{Al}_{2} \mathrm{O}_{3}$ and $\mathrm{Cu}$ nanocrystals (hybrid nanofluid) and the effects of important hydrodynamic parameters on flow character are identified. Parvin and Chamkha ${ }^{19}$ presented an evaluation of naturally convective flow with heat exchange and thermal characteristics in an odd-shaped geometry. The complicated barriers inside solar structures with Newtonian fluid flow is studied by Menni et al. ${ }^{20}$ Some further important results on heat transfer with suspended particles and different geometry can perhaps be found in Refs. [21-28].

The above studies did not consider immiscible flows in which two fluids are separated by an interface and do not mix. Such flows arise in geo-hydrology, nuclear reactor thermo-hydraulics, slurry transport, coating dynamics, petrochemical separation techniques, desalination, and oil extraction systems. Many excellent studies of immiscible hydrodynamics and transport phenomena have therefore been communicated. Bird et al. ${ }^{29}$ reported on the laminar flow of two immiscible fluids in a channel. Umavathi et al. ${ }^{30,31}$ considered polar (couple stress) and Newtonian immiscible fluids in a channel. Kumar et al. ${ }^{32}$ provided an analytical solution for two immiscible (micropolar and Newtonian) fluids in a composite porous medium inside a vertical channel. Srinivas and Murthy ${ }^{33}$ considered immiscible flows of two couple stress fluids in porous layers. Srinivas and Murthy ${ }^{34,35}$ further investigated the influence of entropy generation in heat transfer of two couple stress immiscible fluids. Borrelli et al. ${ }^{36}$ considered the hydromagnetic flow of two immiscible Newtonian fluids in a channel.

One of the primary problems in evaluating the behavior of two immiscible liquid flows is the presence of an unusual interface in the area under consideration. Therefore, the interface of immiscible liquids is important for the design of industrial systems (e.g., chemical separation devices, nuclear reactor transport, etc.). Tauber et al. ${ }^{37}$ addressed the deformations of a shearing immiscible fluid interface. $\mathrm{Yih}^{38}$ found that when the viscosity of one fluid shifts to another, the tangential velocity slope, causing strife at the interface. DeBar ${ }^{39}$ introduced the volume of fluid procedure, which was later improved by Youngs. ${ }^{40} \mathrm{Li}^{41}$ investigated the VOF for interface development between two immiscible liquids. The VOF technique was used by Li and Renardy ${ }^{42}$ to investigate the contact between two immiscible fluids. Vinay and Wachem ${ }^{43}$ investigated the interface development of immiscible fluids including VOF.

Most of the above numerical approaches have proved to be generally reliable for simulating accurately the interface dynamics in immiscible flows. However, both artificial and normal conditions exist where the perspective of time dependence is deeply desirable. There are no real circumstances in immiscible fluid dynamics that do not feature some instability. Consequently, the study of the time-dependent flow of immiscible liquids is critical for providing a deeper appreciation of the actual flow behavior of relevance to for example industrial processes. Despite its actual existence, the time-dependent unstable fluid flow of two immiscible liquids has received relatively sparse 
attention in scientific works. The unstable immiscible flow problem has been examined carefully by Tryggvason et al. ${ }^{44}$ via the front tracking device. Riaz et al. ${ }^{45}$ explored the volatility of the immiscible two-phase flow in porous media. Vajravelu et al. ${ }^{46}$ investigated the magnetohydrodynamic (MHD) flow of immiscible Newtonian liquids in permeable layers. Thermo molded (heat and mass transfer) in immiscible viscous liquid channel flow was explored by Umavathi et al. ${ }^{47}$

A variety of linear and nonlinear fluid flow problems can be numerically solved using finite difference, finite element, and finite volume techniques. Devakar and Raje ${ }^{48}$ numerically explored the time-dependent unsteady flow of two immiscible fluids by the Crank-Nicolson finite difference method. To achieve an acceptable degree of accuracy, the low-order approaches do use several grid points to obtain specific outcomes at such defined points. The differential quadrature approach was proposed by Bellman $^{49}$ to develop an efficient discretization strategy to achieve concise numerical solutions with significantly reduced grid stages. Quan and Chang ${ }^{50}$ improved the DQM approach. Various test functions, namely, Lagrange interpolation polynomials, sink and spline, cubic and modifiedcubic B-spline are used in Ref. [51] for the calculation of weighting coefficients in DQM. This technique is both easily applicable and efficient strategically, contributing to error reduction and simple to enforce as described by Katta et al. ${ }^{52}$ Katta and Joshi ${ }^{34}$ used DQM to numerically analyze the hydromagnetic flow of a viscoelastic Jeffrey fluid in a porous medium. ${ }^{53}$

Previous studies have been confined to examining the smooth and unstable movement of the micropolar fluid, the steady stream of two immiscible fluids, and interface monitoring through different modes. In this paper, we extend the earlier studies to consider the unsteady flow of two immiscible Eringen micropolar and Newtonian fluids with a moving interface in the horizontal channel. The microrotation parameter affects the entire domain. First, the micro-rotation profile is computed, and then interface tracking with either constant, periodic, or decaying applied pressure gradients are obtained numerically by solving the coupled partial differential equations using the modified cubic B-spline differential quadrature method (MCB-DQM) to examine the effects of time, amplitude, wavenumber on the interface evolution and the velocity and micro-rotation velocity fields. The present work finds applications in flow control and separation processes in chemical engineering. ${ }^{8}$

\section{GOVERNING EQUATION}

The micropolar fluid flow field equations are: ${ }^{1}$

$$
\begin{aligned}
& \rho_{t}+\nabla \cdot(\rho u)=0 \\
& \rho f-\nabla p+\delta \nabla \times \omega-(\mu+\delta) \nabla \times \nabla \times u
\end{aligned}
$$

$$
\begin{gathered}
+\left(\gamma_{1}+2 \mu+\delta\right) \nabla(\nabla \cdot u)-\rho \cdot \frac{\partial u}{\partial t}=0 \\
\rho \xi-2 \delta \omega+\delta \nabla \times v-\lambda_{1} \nabla \times \nabla \times \omega \\
+\left(\lambda_{1}+\lambda_{2}+\lambda_{3}\right) \nabla(\nabla \cdot \omega)-\rho \cdot \tau \frac{\partial \omega}{\partial t}=0
\end{gathered}
$$

The descriptions of notations and symbols $\lambda_{1}, \lambda_{2}, \lambda_{3}$, $\gamma_{1}, \mu, \delta, u, \omega, f, \xi, \rho, \tau$, and $p$ are being mentioned in the nomenclature section. As Newtonian fluid does not contain any micro rotational effect the Eq. (2) is reduced as

$$
\rho \cdot \frac{\partial u}{\partial t}=\rho \mu-\nabla p-\mu \nabla \times \nabla \times u+\left(\gamma_{1}+2 \mu\right) \nabla(\nabla \cdot u)
$$

\subsection{The Volume of Fluid Method}

In the system of two immiscible liquids, the characteristics resulting in the momentum model are defined by each control volume. The following equations normalize the average density and viscosity quantities: ${ }^{42}$

$$
\begin{gathered}
\rho^{*}=\rho_{1}+C_{s}\left(\rho_{2}-\rho_{1}\right) \\
\mu^{*}=\mu_{1}+C_{s}\left(\mu_{2}-\mu_{1}\right)
\end{gathered}
$$

Here $\rho_{1}, \mu_{1}$ be the density and viscosity of micropolar fluid and $\rho_{2}, \mu_{2}$ be the density and viscosity of Newtonian fluid and $C_{s}$ is volume fraction function. The interface tracking function $C_{i}$ is transported by the velocity field $u^{*}: 42,54202111: 44: 4$

$$
\text { Sy Ingenta. } \frac{\partial C_{i}}{\partial t}+u^{*} \cdot \nabla C_{i}=0
$$

The flow velocity is driven by a single mass transfer equation: ${ }^{42}$ with the resultant velocity field shared between the transitions.

$$
\rho^{*}\left(\frac{\partial u^{*}}{\partial t}+u^{*} . \nabla u^{*}\right)=-\nabla p+\mu^{*} \nabla^{2} u^{*}+\rho^{*} g+f
$$

Here $g$ is the gravitational acceleration, $f$ is the body force per unit mass, and $-\nabla \rho$ is the applied pressure term. The characteristics of the velocity equation are calculated with increasing governing volume in a two-phase system.

\section{FORMULATION}

Consider the unsteady, laminar, and unidirectional flow of two immiscible, micropolar, and Newtonian fluids undergoing shearing motions. Owing to viscosity jump primarily, an interfacial instability occurs. Both fluids are viscous and incompressible and move between two horizontal parallel non-porous plates. The plates are located in the $X-Z$ plane as depicted in Figure 1. The maximum flow velocity is $U_{0}$ in the $x$ direction. Let $\rho_{1}, \mu_{1}$ be the density and viscosity of micropolar fluid which lies in $(-k \leq y \leq$ $l_{1}$ ) with the interface height $l_{1}$. The Newtonian fluid has the density $\rho_{2}$ and the viscosity $\mu_{2}$ occupies in $\left(l_{1} \leq y \leq k\right)$. The evolution of the interface is analysed by using horizontal attenuation in the initial interface phase. Let the initial interface be an Eigen-mode (In an oscillating interface, 


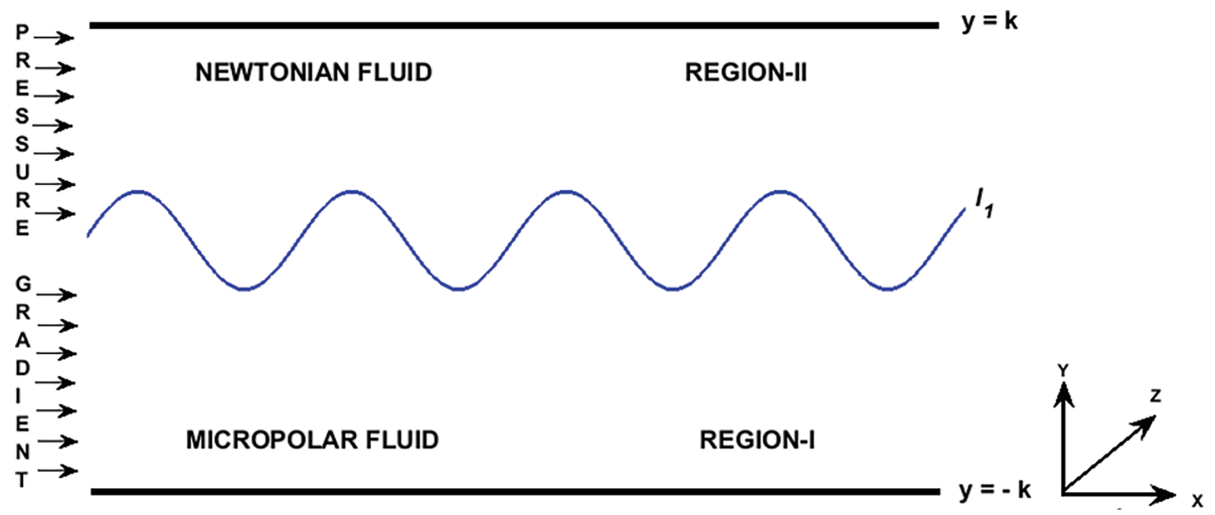

Fig. 1. Geometrical configuration of the Mean flow of two immiscible fluids.

a normal mode is one in which all elements of the system operate at the same frequency.), say $l_{1} y_{m}+A_{0} \cos \left(B_{0} y\right)$ where $y_{m}$ is the average depth of interface $A_{0}, B_{0}$ are the amplitude and wavenumber. The flow of the immiscible fluids is mobilized by $x$-direction applied common pressure gradient (either constant, decaying, or periodic). The average density ${ }^{42,54} \rho^{*}=\rho_{1}+C_{s}\left(\rho_{2}-\rho_{1}\right)$ and viscosity $\mu^{*}=\mu_{1}+C_{s}\left(\mu_{2}-\mu_{1}\right)$.

The body force $f$ is modeled as surface tension force by the continuum surface force model, and the surface tension is reconstructed as a volume force. ${ }^{42}$

$$
F_{s}=\sigma \varsigma \nabla C_{i}
$$

Here $\sigma$ is the interfacial tension between two liquid phases and $s$ is the mean free surface curvature, given by $s=-\left(\nabla^{2} C_{i}\right) /\left(\left\|\nabla C_{i}\right\|\right)$. Since the flow is assumed as unidirectional so $\nabla C_{i}=\left(\partial C_{i} / \partial y\right)$ and $\nabla^{2} C_{i}=\left(\partial^{2} C_{i} / \partial y^{2}\right)$. Hence Eq. (9) can be written as

$$
F_{s}=\sigma \frac{\partial^{2} C_{i}}{\partial y^{2}}
$$

The micropolar fluid velocity $u_{1}$, Newtonian liquid velocity $u_{2}$, micro-rotation vector $\omega$ in the region $\mathrm{I}$, mean flow velocity $u^{*}$, and volume fraction $C_{i}$ are mathematically denoted as $u_{1}=\left(u_{1}(y, t), 0,0\right), u_{2}=$ $\left(u_{2}(y, t), 0,0\right), \omega=\left(0,0, C_{*}(y, t)\right), u^{*}=\left(u^{*}(y, t), 0,0\right)$, $C_{i}=\left(C_{i}(y, t), 0,0\right)$ respectively. The two-fluid flow is unstable, incompressible $\nabla \cdot u_{1}=0, \nabla \cdot u_{2}=0$ and induced by an $X$-direction pressure gradient and the microrotation vector is present only in the region-I fluid. Given this, Eqs. (2)-(3) can then be simplified to:

Region-I for Micro-polar fluid $\left(-k \leq y \leq l_{1}\right)$

$$
\begin{array}{r}
\rho_{1} \frac{\partial u_{1}}{\partial t}=-\frac{\partial p}{\partial x}+\delta \frac{\partial C_{*}}{\partial y}+\left(\alpha_{1}+\delta\right) \frac{\partial^{2} u_{1}}{\partial y^{2}} \\
\rho_{1} \tau \frac{\partial C_{*}}{\partial t}=\lambda_{1} \frac{\partial^{2} C_{*}}{\partial y^{2}}-\delta\left(2 C_{*}+\frac{\partial u_{1}}{\partial y}\right)
\end{array}
$$

Region-II for Newtonian fluid $\left(l_{1} \leq y \leq k\right)$

$$
\rho_{2} \frac{\partial u_{2}}{\partial t}=\alpha_{2} \frac{\partial^{2} u_{2}}{\partial y^{2}}-\frac{\partial p}{\partial x}
$$

By an exchange in momentum, the fluid layers are mechanically linked by the system. We do allow for the possibility of a jump across the interface. The interface movement of immiscible fluids of various densities and viscosity is described by the volume fraction function $C_{s}$ and three conditions are used to monitor the interface. ${ }^{42}$ Each node in the computing grid records the volume fraction of micropolar and Newtonian fluid. The concentration function is the volume fraction field at the discrete level. If a cell is occupied with the micropolar fluid then $C_{s}=0$. The cell does not contain any volume of micropolar fluid and occupied with Newtonian fluid as $C_{s}=1$. There is a fluid interface in the cell, then $0<C_{s}<1$. As per the specific value of the $C_{s}=0.5$, We could obtain a unique volume fraction field from an interface. Hence density value $\rho^{*}=\rho_{1}+0.5\left(\rho_{2}-\rho_{1}\right)=\left(\left(\rho_{2}+\rho_{1}\right)\right) / 2=$ and viscosity value $\mu^{*}=\mu_{1}+0.5\left(\mu_{2}-\mu_{1)}=\left(\mu_{2}+\mu_{1}\right) / 2\right.$ allows jump across the interface. If $C_{s}=0$ and 1 then there no penetration of micropolar and Newtonian fluids in each other region.

Hence by using the Eqs. (2)-(9), the mean flow in the region $(-k \leq y \leq k)$ and interface track in $(-k \leq y \leq k)$ are governed by the following equations:

$$
\begin{aligned}
& \frac{\partial C_{i}}{\partial t}+u^{*} \frac{\partial C_{i}}{\partial y}=0 \\
& \rho^{*} \frac{\partial u^{*}}{\partial t}=-\frac{\partial p}{\partial x}+\left(\mu^{*}+\delta\right) \frac{\partial^{2} u^{*}}{\partial y^{2}}+\delta \frac{\partial C_{*}}{\partial y}+\rho^{*} g+\sigma \frac{\partial^{2} C_{i}}{\partial y^{2}}
\end{aligned}
$$

The classical no-slip and hyper-stick conditions are considered at the boundaries. These conditions can be written mathematically as follows.

Initial conditions: At $t \leq 0$,

$$
\begin{aligned}
& u_{1}(y, t)=u^{*}(y, t)=C_{*}(y, t)=0, \quad \text { and } \\
& C_{i}(y, t)=l_{1} y_{m}+A_{0} \cos \left(B_{0} y\right)
\end{aligned}
$$

Boundary conditions: At $t<0$,

$$
u_{1}(-k, t)=u^{*}(-k, t)=C_{*}(-k, t)=u^{*}(k, t)=0,
$$




$$
u_{1}\left(l_{1}, t\right)=\varepsilon, \quad C_{*}\left(l_{1}, t\right)=-\frac{1}{2} \frac{\partial u_{1}}{\partial t}
$$

It is judicious to introduce the following nondimensional parameters $\bar{x}=(x / k), \bar{y}=(y / k), \overline{u^{*}}=$ $\left(u^{*} / U_{0}\right), \overline{u_{P}}=\left(u_{P} / U_{0}\right), \bar{p}=\left(p / p^{*} U_{0}^{2}\right), \bar{t}=\left(t U_{0} / k\right) \varepsilon \in$ $\left[-A_{0}, A_{0}\right]$ s.t $\sqrt{\left(u_{1}(\varepsilon, t)-c_{i}\right)^{2}}=0, \lambda_{1}=\left(\mu_{1}+\delta / 2\right) \tau$ with $\tau=k^{2}$. Also, the following dimensionless numbers are invoked: Eringen micropolar parameter $n_{1}=\left(\delta / \mu_{1}\right), \operatorname{Re}=$ $\left(\rho_{1} U_{0} / \mu_{1}\right)$ is the Reynolds number, $\operatorname{Fr}=\left(U_{0}{ }^{2}\right) / g k$ is the Froude number. $r_{1}=\left(\mu_{2} / \mu_{1}\right)$ is the ratio of viscosity, $r_{2}=$ $\left(\rho_{2} / \rho_{1}\right)$ is the ratio density and $C a=\left(\mu^{*} U_{0}\right) / \sigma$ is the capillary number. The pressure variation based on time is implemented from $X$-axis for momentum in the mean flow. Three different cases for $G e(t)-\nabla \rho=-\partial p / \partial x$ with $t>0$ are applied to obtain numerical results.

I: $G e(t)=G e($ constant $)$

II: $G e(t)=G e * \operatorname{Sin}(w t)$ (periodic with parameter $\mathrm{w})$

III: $G e(t)=G e * e^{-\lambda t}$ (decaying with parameter $\lambda$ ).

After dropping the bars and introducing the above nondimensional parameters, appropriate initial, interfacial, and boundary conditions according to the scheme, the equations can be reduced to:

Region-I $(-k \leq y \leq 0)$ (Micro-polar fluid region):

$$
\begin{aligned}
\frac{\partial u_{1}}{\partial t} & =G e(t)+\frac{n_{1}}{R e} \frac{\partial C_{*}}{\partial y}+\frac{\left(n_{1}+1\right)}{R e} \frac{\partial^{2} u_{1}}{\partial y^{2}} \\
\frac{\partial C_{*}}{\partial t} & =\frac{\left(\left(n_{1} / 2\right)+1\right)}{\operatorname{Re}} \frac{\partial^{2} C_{*}}{\partial y^{2}}-\frac{n_{1}\left(2 C_{*}+\left(\partial u_{1} / \partial y\right)\right)}{\operatorname{Re}}
\end{aligned}
$$

The mean flow in the region $(-k \leq y \leq k)$, and interface track $(-k \leq y \leq k)$ are governed by:

$$
\begin{aligned}
\frac{\partial C_{i}}{\partial t}+u^{*} \frac{\partial C_{i}}{\partial y}= & 0 \\
\frac{\partial u^{*}}{\partial t}= & \frac{G e(t)}{\left(1+C_{s}\left(r_{2}-1\right)\right)} \\
& +\frac{\left(n_{1}+1\right)\left(1+C_{s}\left(r_{1}-1\right)\right)}{\operatorname{Re}\left(1+C_{s}\left(r_{2}-1\right)\right)} \frac{\partial^{2} u^{*}}{\partial y^{2}}
\end{aligned}
$$

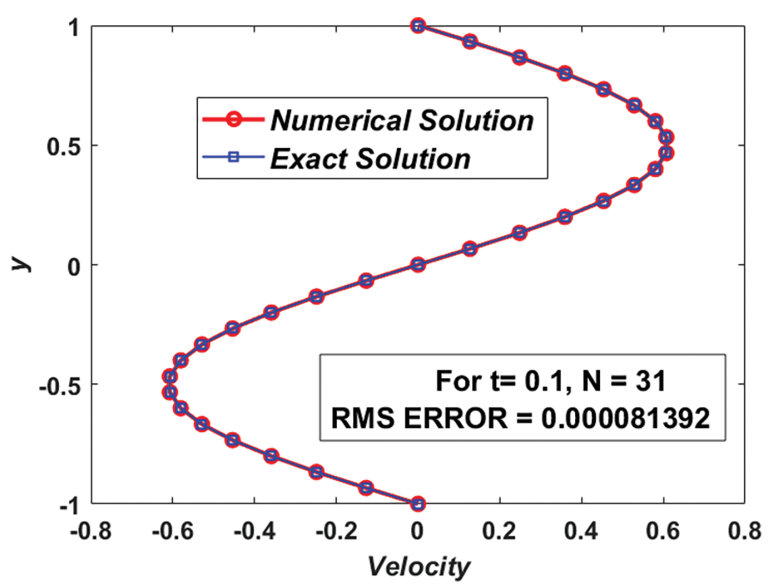

Fig. 2. The velocity profile comparison at time $t=0.1$ and $N=31$.

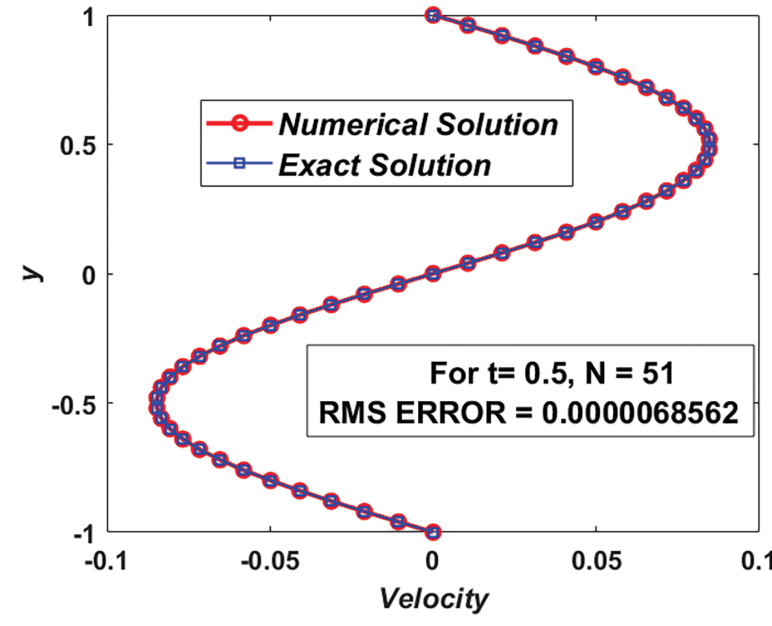

Fig. 3. The velocity profile comparison at time $t=0.5$ and $N=51$.

$$
\begin{aligned}
& +\frac{n_{1}\left(1+C_{s}\left(r_{1}-1\right)\right)}{\operatorname{Re}\left(1+C_{s}\left(r_{2}-1\right)\right)} \frac{\partial C_{*}}{\partial y}+\frac{1}{F r} \\
& -\frac{1+C_{s}\left(r_{1}-1\right)}{\operatorname{Re}^{*} C a\left(1+C_{s}\left(r_{2}-1\right)\right)} \frac{\partial^{2} C_{i}}{\partial y^{2}}
\end{aligned}
$$

Equations (17), (18) are considered as initial and boundary conditions with $k=1$.

\section{NUMERICAL SOLUTION WITH MCB-DQM SCHEME}

The differential quadrature technique is effective in the simulation of various linear and nonlinear coupled partial differential equations. The computational accuracy of DQM is very good and only a few grid points and less time are required to achieve high precision. In this problem, we started with 31 points and observe a good agreement in the computed and exact result. The DQ method's strength lies in its ability to handle a wide range of fluid boundary conditions.

To analyze the interface tracking between micropolar and Newtonian fluids, first, the microrotation vector is

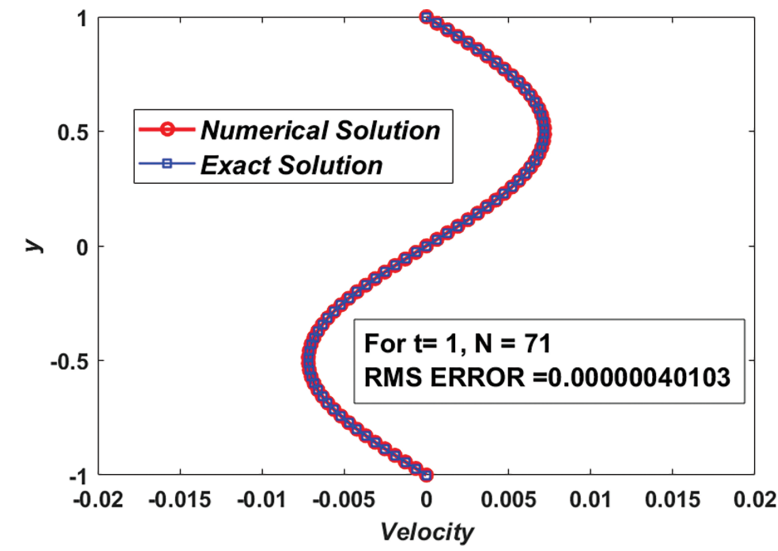

Fig. 4. The velocity profile comparison at time $t=1$ and $N=71$. 


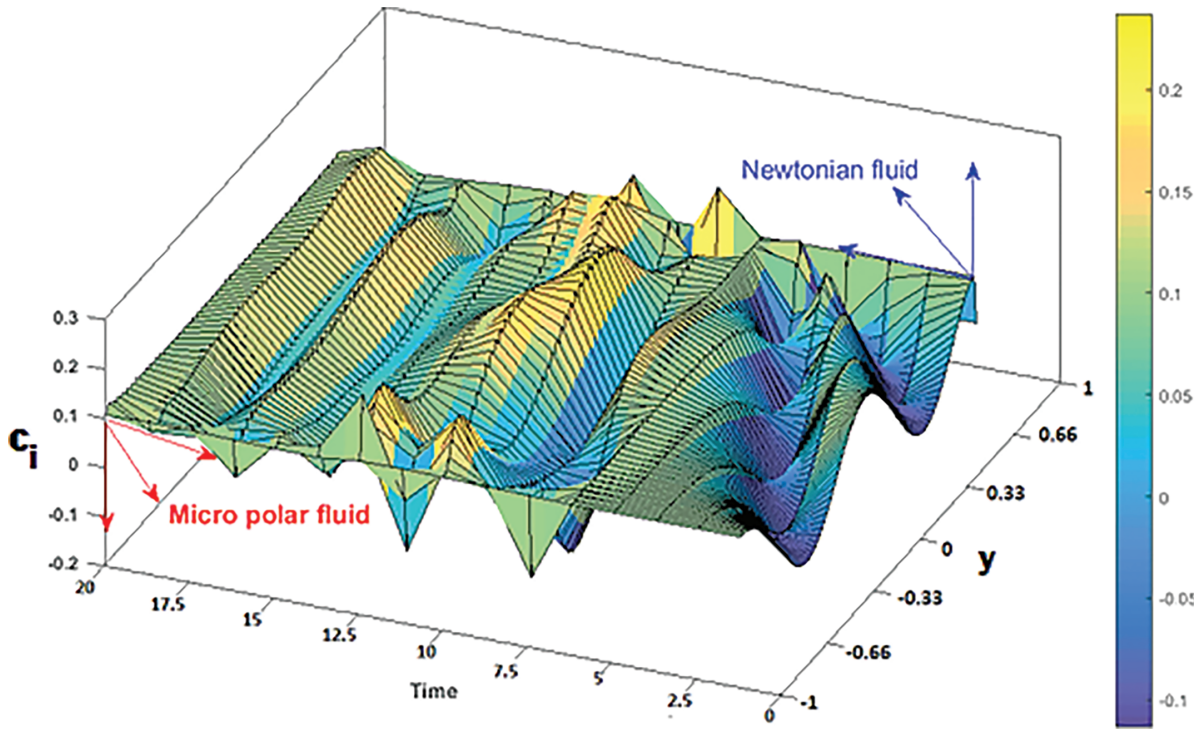

Fig. 5. 3D evolution of an interface between Micropolar and Newtonian fluids with time.

calculated in the domain $(-1 \leq y \leq 0)$ using the velocity vector of micropolar fluid, and then the flow velocity and interface vector in $(-1 \leq y \leq 1)$ is obtained. The domains $[-1,0]$ for microrotation vector and $[-1,1]$ for flow velocity and interface vector are equally discretized with phase length $h$ in the spline-based differential quadrature method ${ }^{52,55}$ with phase length h: The nodes are placed with uniform step size $h$.

$$
\begin{aligned}
& a=y_{1}<y_{2}<\cdots<y_{n-1}<y_{N}=b, \text { such that } \\
& y_{i+1}-y_{i}=h \text { on the real axis }
\end{aligned}
$$

After that, the approximation for $1 \mathrm{st}$ and 2 nd order derivatives of $\boldsymbol{u}_{\boldsymbol{I}}(\boldsymbol{y}, \boldsymbol{t}), \boldsymbol{C}_{*}(\boldsymbol{y}, \boldsymbol{t}), \boldsymbol{u}^{*}(\boldsymbol{y}, \boldsymbol{t})$, and $\boldsymbol{C}_{\boldsymbol{i}}(\boldsymbol{y}, \boldsymbol{t})$ are obtained at any time on the nodes $\boldsymbol{y}_{\boldsymbol{i}}$ as-

For $i=1,2,3, \ldots, N$ and $j=1,2, \ldots, N$

$$
\begin{aligned}
& \frac{\partial u_{1}}{\partial \mathrm{y}}=\sum_{j=1}^{N} Z_{i, j}^{(1)} u_{1}\left(y_{j}, t\right) \\
& \frac{\partial^{2} u_{1}}{\partial \mathrm{y}^{2}}=\sum_{j=1}^{N} Z_{i, j}^{(2)} u_{1}\left(y_{j}, t\right) \\
& \frac{\partial C_{*}}{\partial \mathrm{y}}=\sum_{j=1}^{N} Z_{i, j}^{(1)} C_{*}\left(y_{j}, t\right) \\
& \frac{\partial^{2} C_{*}}{\partial \mathrm{y}^{2}}=\sum_{j=1}^{N} Z_{i, j}^{(2)} C_{*}\left(y_{j}, t\right) \\
& \frac{\partial u^{*}}{\partial \mathrm{y}}=\sum_{j=1}^{N} Z_{i, j}^{(1)} u^{*}\left(y_{j}, t\right) \\
& \frac{\partial^{2} u^{*}}{\partial \mathrm{y}^{2}}=\sum_{j=1}^{N} Z_{i, j}^{(2)} u^{*}\left(y_{j}, t\right) \\
& \frac{\partial C_{*}}{\partial \mathrm{y}}=\sum_{j=1}^{N} Z_{i, j}^{(1)} C_{*}\left(y_{j}, t\right)
\end{aligned}
$$

Here $Z_{i, j}^{(1)} Z_{i, j}^{(2)}$ are the weighting coefficients of 1 st and 2nd order derivatives ${ }^{52,53}$ for $\mathrm{n}$ discrete knots $\left(y_{1}, y_{2}, \ldots y_{n}\right)$ derived from the updated $\mathrm{B}$-spline functions. The cubic B-spline at the knots is defined as ${ }^{52}$
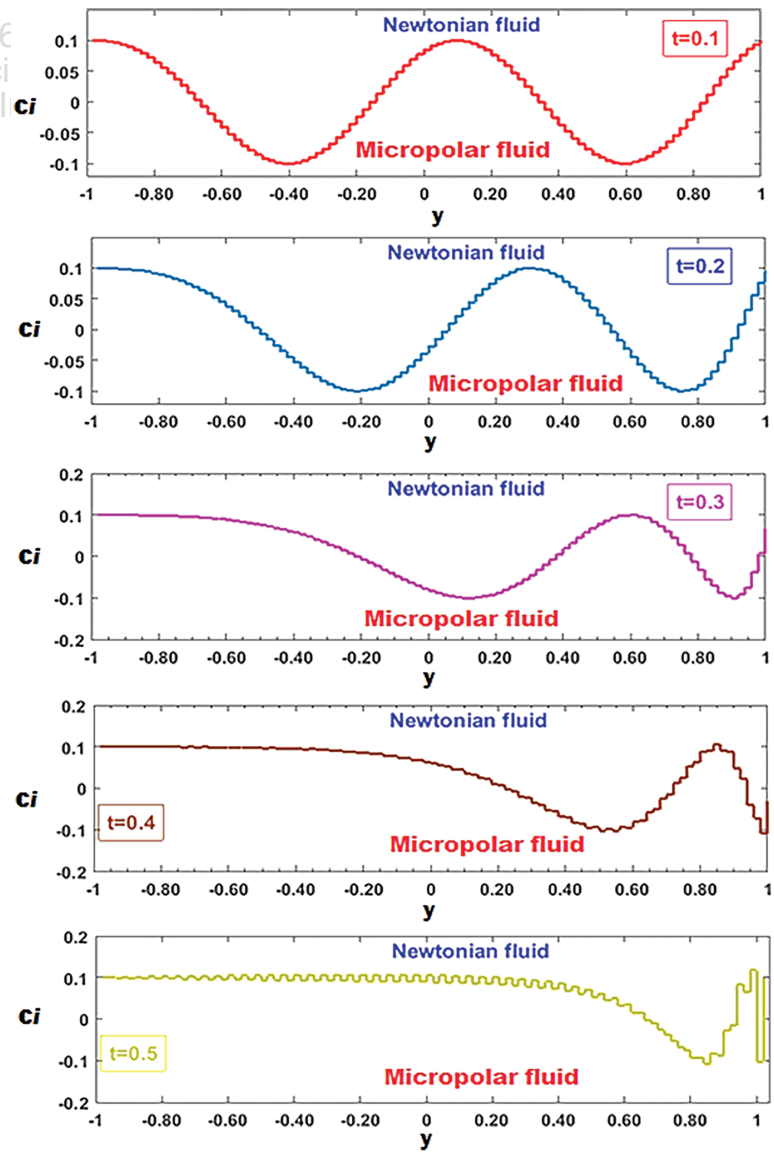

Fig. 6. Interface tracking with varying small time when $G e=10, R e=$ $2, \boldsymbol{r}_{\mathbf{1}}=0.5, \boldsymbol{r}_{\mathbf{2}}=0.5, \boldsymbol{C}_{s}=0.5, C a=0.5, F r=\mathbf{2}, \boldsymbol{n}_{\mathbf{1}}=\mathbf{0 . 5}$. 
$\chi_{j}^{\prime}(y)=\frac{1}{h^{3}} \begin{cases}\left(y-y_{j-2}\right)^{3}, & y \epsilon\left[y_{j-2}, y_{j-1}\right) \\ \left(y-y_{j-2}\right)^{3}-4\left(y-y_{j-1}\right)^{3}, & y \epsilon\left[y_{j-1}, y_{j}\right) \\ \left(y_{j+2}-y\right)^{3}-4\left(y_{j+1}-y\right)^{3}, & y \epsilon\left[y_{j}, y_{j+1}\right) \\ \left(y_{j+2}-y\right)^{3}, & y \epsilon\left[y_{j+1}, y_{j+2}\right) \\ 0, & \text { otherwise }\end{cases}$

where $\left\{\chi_{0}(y), \chi_{1}(y), \chi_{2}(y) \ldots, \chi_{n+1}(y)\right\}$ are the basis function over the region $[a, b]$. The basis function is updated as follows to get a system of equations that could be expressed by a diagonal-dominated matrix. ${ }^{52,53}$

$$
\left.\begin{array}{l}
D_{1}(y)=\chi_{1}(y)+2 \chi_{0}(y) \\
D_{2}(y)=\chi_{2}(y)-\chi_{0}(y) \\
D_{j}(y)=\chi_{j}, \text { for } \quad j=3, \ldots N-2 \\
D_{N-1}(y)=\chi_{N-1}(y)-\chi_{N+1}(y) \\
D_{N}(y)=\chi_{N}(y)+2 \chi_{N+1}(y)
\end{array}\right\}
$$
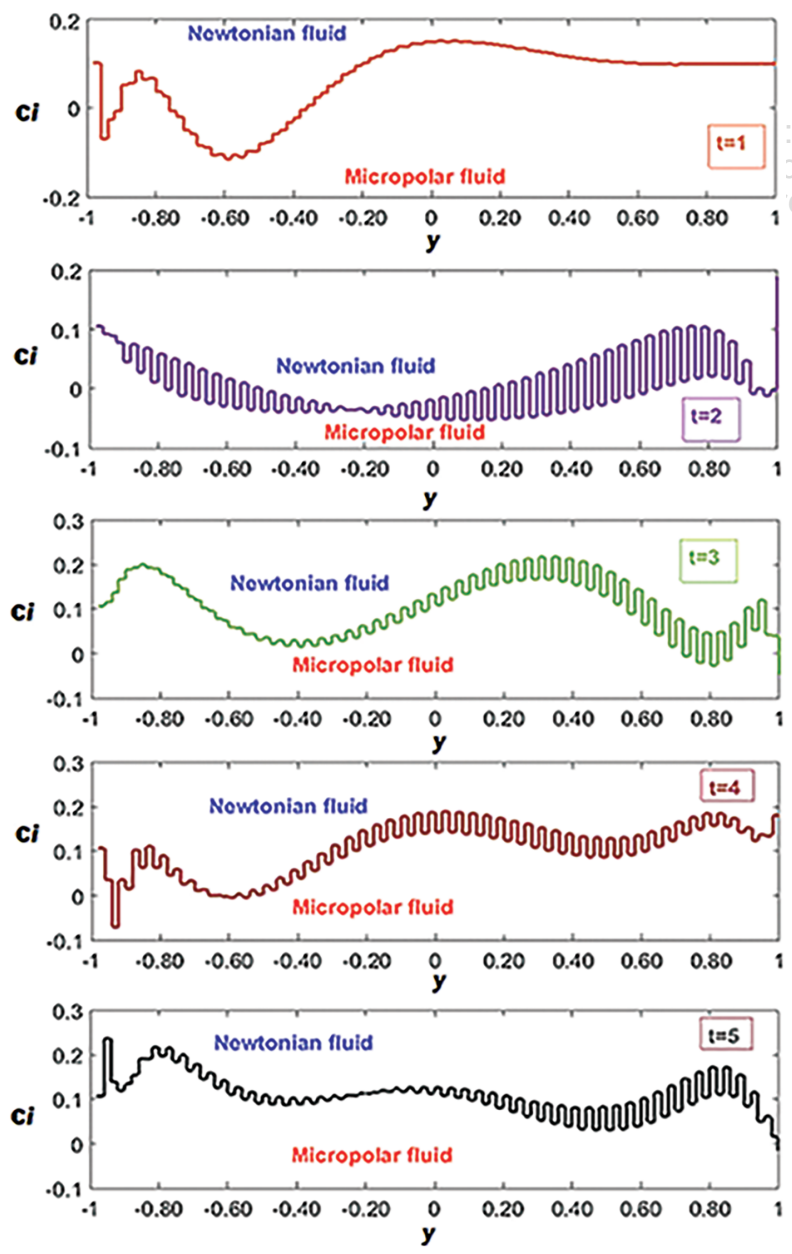

Fig. 7. Interface tracking with varying time when $G e=10, R e=2$, $\boldsymbol{r}_{1}=0.5, \boldsymbol{r}_{2}=0.5, \boldsymbol{C}_{s}=0.5, \boldsymbol{C a}=0.5, \boldsymbol{F r}=\mathbf{2}, \boldsymbol{n}_{1}=\mathbf{0 . 5}$.
The derivatives of the basic functions are as follows:

$\chi_{j}^{\prime}(y)$

$$
=\frac{1}{h^{3}}\left\{\begin{array}{cc}
3\left(y-y_{j-2}\right)^{2}, & y \in\left[y_{j-2}, y_{j-1}\right) \\
3\left(y-y_{j-2}\right)^{2} & y \in\left[y_{j-1}, y_{j}\right) \\
-12\left(y-y_{j-1}\right)^{2}, & \\
-3\left(y_{j+2}-y\right)^{2} & y \in\left[y_{j}, y_{j+1}\right) \\
+12\left(y_{j+1}-y\right)^{2}, & \\
-3\left(y_{j+2}-y\right)^{2}, & y \in\left[y_{j+1}, y_{j+2}\right) \\
0, & \text { otherwise }
\end{array}\right.
$$

$$
\begin{aligned}
& D_{1}^{\prime}(y)=\chi_{1}^{\prime}(y)+2 \chi_{0}^{\prime}(y) \\
& D_{2}^{\prime}(y)=\chi_{2}^{\prime}(y)-\chi_{0}^{\prime}(y) \\
& D^{\prime}{ }_{j}(y)=\chi_{j}^{\prime}, \quad \text { for } \quad j=3, \ldots . . N-2 \\
& D^{\prime^{\prime}}{ }_{N-1}(y)=\chi_{N-1}^{\prime}(y)-\chi_{N+1}^{\prime}(y) \\
& D^{\prime}{ }_{N}(y)=\chi^{\prime}{ }_{N}(y)+2 \chi^{\prime}{ }_{N+1}(y)
\end{aligned}
$$
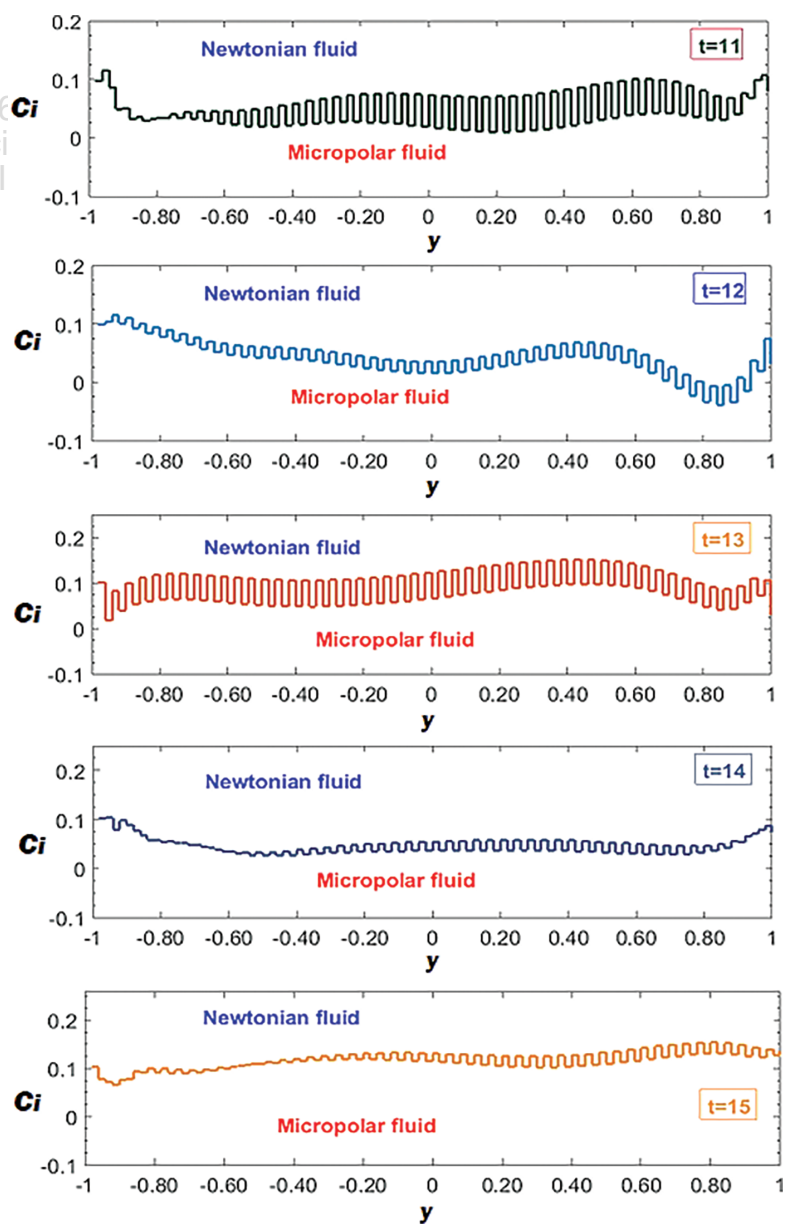

Fig. 8. Interface tracking with varying high time when $G e=10, R e=$ $2, \boldsymbol{r}_{1}=0.5, \boldsymbol{r}_{2}=0.5, \boldsymbol{C}_{s}=0.5, \boldsymbol{C a}=0.5, \boldsymbol{F r}=\mathbf{2}, \boldsymbol{n}_{1}=\mathbf{0 . 5}$. 

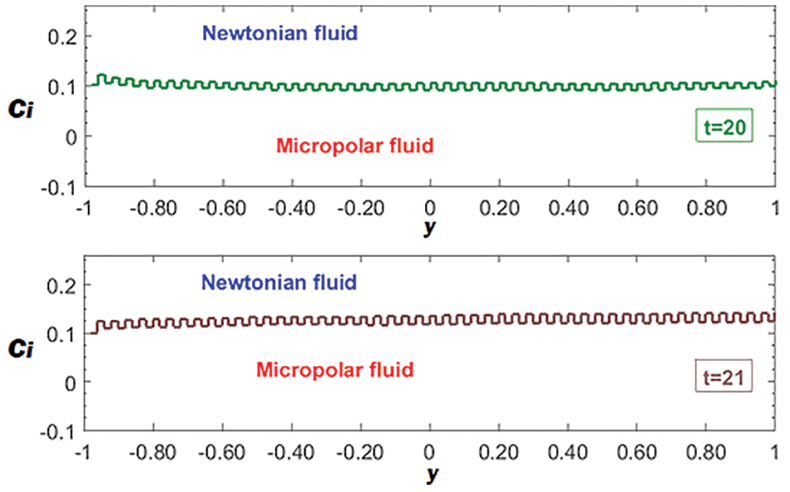

Fig. 9. Interface tracking with varying high time when $G e=10, R e=$ 2, $\boldsymbol{r}_{1}=0.5, \boldsymbol{r}_{2}=0.5, \boldsymbol{C}_{s}=0.5, \boldsymbol{C a}=0.5, \boldsymbol{F r}=\mathbf{2}, \boldsymbol{n}_{1}=\mathbf{0 . 5}$.

The estimate of the first-order derivative is

$$
D_{k}^{\prime}\left(y_{i}\right)=\sum_{j=1}^{N} Z_{i, j}^{(1)} \chi_{k}\left(y_{j}\right) \begin{array}{r}
\text { for } \quad i=1,2, \ldots, N \\
k=1,2, \ldots, N
\end{array}
$$

Then using $Z_{i, j}^{(1)}$ for $i=1,2, \ldots, N$ in Eq. (19) the following tri-diagonal system of equations is established as:

$$
\times\left[\begin{array}{c}
Z_{i, 1}^{(1)} \\
Z_{i, 2}^{(1)} \\
Z_{i, 3}^{(1)} \\
\cdot \\
\cdot \\
\cdot \\
\cdot \\
Z_{i, N-1}^{(1)} \\
Z_{i, N}^{(1)}
\end{array}\right]=\left[\begin{array}{c}
D_{1}^{\prime}{ }_{1}\left(y_{i}\right) \\
D_{2}^{\prime}\left(y_{i}\right) \\
D^{\prime}{ }_{3}\left(y_{i}\right) \\
\cdot \\
\cdot \\
\cdot \\
\cdot \\
D_{N-1}^{\prime}\left(y_{i}\right) \\
D_{N}^{\prime}\left(y_{i}\right)
\end{array}\right]
$$

Solving the above system the weighting coefficients $\left\{Z_{1,1}^{(1)}, \quad Z_{1,2}^{(1)} \ldots, Z_{i, N}^{(1)}\right\}, \quad\left\{Z_{2,1}^{(1)}, Z_{2,2}^{(1)} \ldots, Z_{2, N}^{(1)}\right\}, \ldots,\left\{Z_{N, 1}^{(1)}\right.$, $\left.Z_{N, 2}^{(1)} \ldots, Z_{N, N}^{(1)}\right\}$ of 1 st order derivatives of linear and angular velocities are obtained and then the value of $Z_{i, j}^{(2)}$ for $i=1,2,3 \ldots N, j=1,2,3 \ldots N$ is calculated as follows

$$
\left.\begin{array}{ll}
Z_{i, j}^{(2)}=2 Z_{i, j}^{(1)}\left(Z_{i, j}^{(1)}-\frac{1}{y_{i}-y_{j}}\right) & \text { for } i \neq j \\
Z_{i, i}^{(2)}=-\sum_{i \neq 1, i \neq j}^{N} Z_{i, j}^{(2)} & i=j
\end{array}\right\}
$$

Substituting the estimation of the 1 st and 2 nd order derivatives by the MCB-DQM scheme, Eqs. (19)-(23) can be written as:

Region-I $(-k \leq y \leq 0)$ (Micro-polar fluid region):

$$
\begin{aligned}
u_{1 t}= & G e(t)+\frac{n_{1}}{\operatorname{Re}}\left(\sum_{j=1}^{N} Z_{i, j}^{(1)} C_{*}\left(y_{j}, t\right)\right) \\
& +\frac{\left(n_{1}+1\right)}{\operatorname{Re}}\left(\sum_{j=1}^{N} Z_{i, j}^{(2)} u_{1}\left(y_{j}, t\right)\right)
\end{aligned}
$$

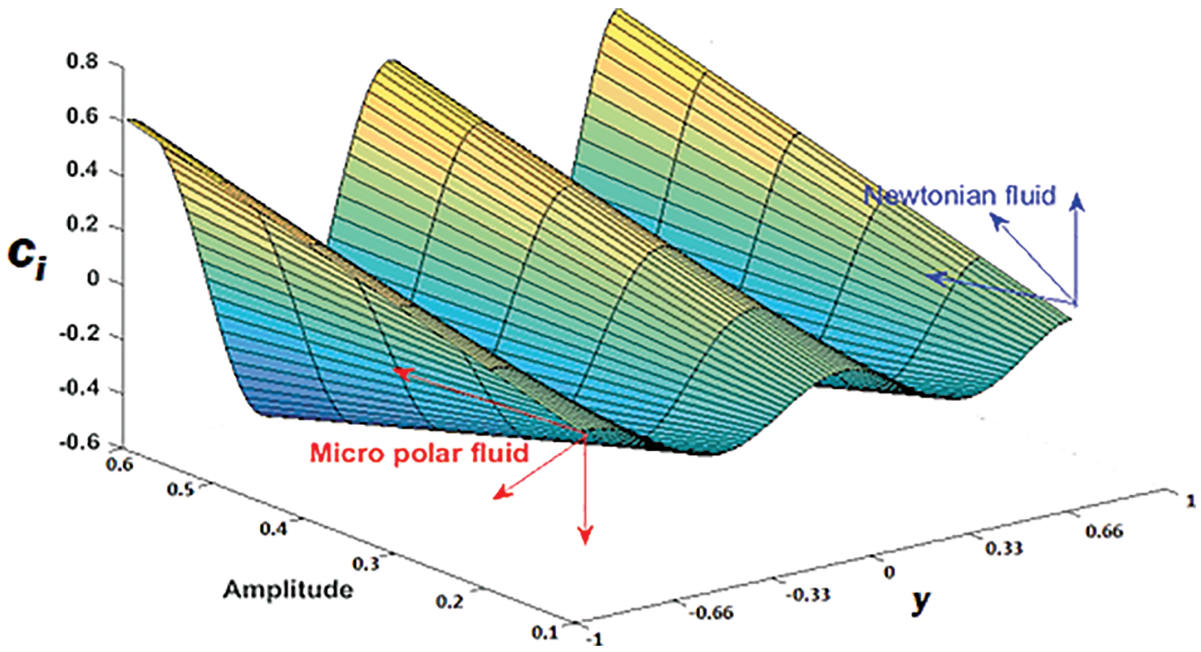

Fig. 10. 3D evolution of an interface between Micropolar and Newtonian fluids with amplitude. 


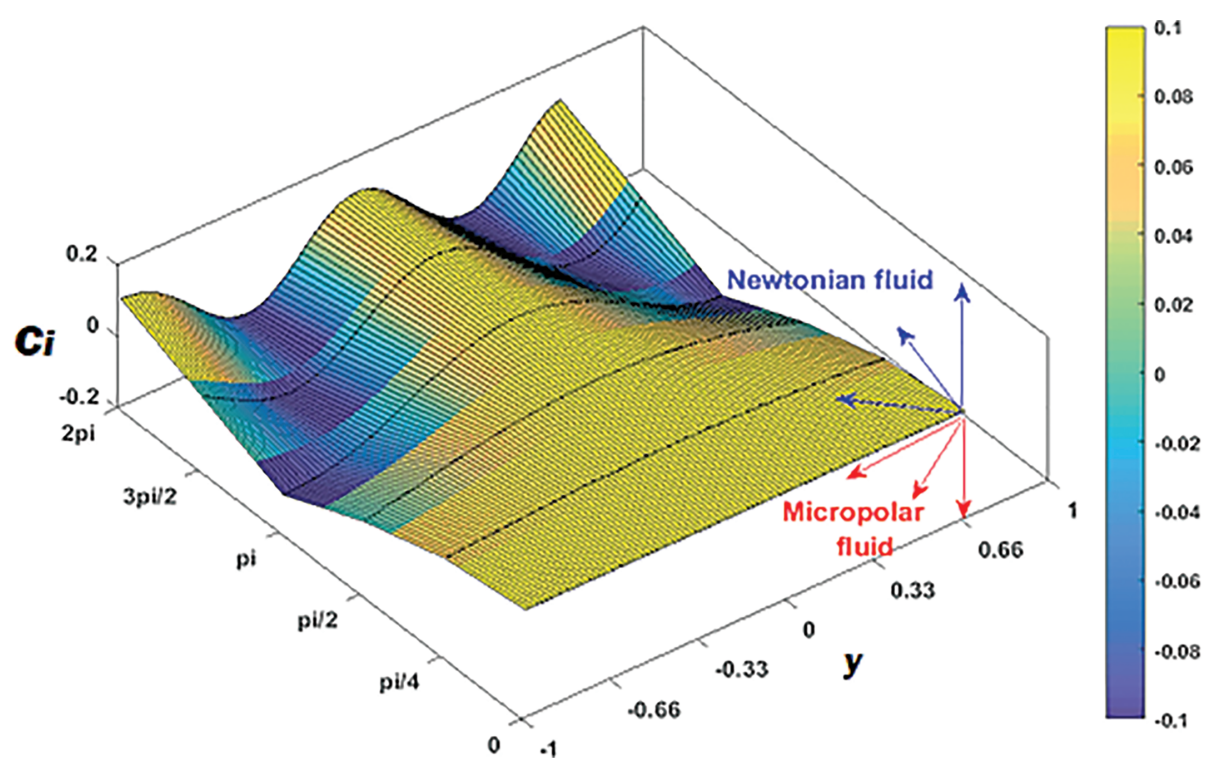

Fig. 11. 3D evolution of an interface between Micropolar and Newtonian fluids with varying wavenumber.

$$
\begin{aligned}
C_{* t}= & \frac{\left(n_{1}+2\right)}{2 \operatorname{Re}}\left(\sum_{j=1}^{N} Z_{i, j}^{(2)} C_{*}\left(y_{j}, t\right)\right) \\
& -\frac{n_{1}}{\operatorname{Re}}\left(2 C_{*}+\sum_{j=1}^{N} Z_{i, j}^{(1)} u_{1}\left(y_{j}, t\right)\right)
\end{aligned}
$$

$$
\begin{array}{r}
U_{2}=U_{1}+\frac{\Delta t}{2} * R\left(u_{11}, C_{* 1}, C_{i 1}, u_{1}^{*}\right) \\
U_{3}=\frac{{ }_{2} U_{0}}{3}+\frac{U_{1}}{3}+\frac{\Delta t}{6} * R\left(u_{12}, C_{* 2}, C_{i 2}, u_{2}^{*}\right) \\
2021 U_{M}=U_{3}+\frac{\Delta t}{2} * R\left(u_{13}, C_{* 3}, C_{i 3}, u_{3}^{*}\right) \\
\text { c Publisters }
\end{array}
$$

In the region $(-k \leq y \leq k)$ :

$$
\begin{aligned}
C_{i t}= & -u^{*}\left(y_{j}, t\right)\left(\sum_{j=1}^{N} Z_{i, j}^{(1)} C_{i}\left(y_{j}, t\right)\right) \\
u^{*}{ }_{t}= & \frac{G e(t)}{r_{2}} \\
& +\frac{\left(n_{1}+1\right)\left(1+C_{s}\left(r_{1}-1\right)\right)}{\operatorname{Re}\left(1+C_{s}\left(r_{2}-1\right)\right)}\left(\sum_{j=1}^{N} Z_{i, j}^{(2)} u^{*}\left(y_{j}, t\right)\right) \\
& +\frac{n_{1}\left(1+C_{s}\left(r_{1}-1\right)\right)}{\operatorname{Re}\left(1+C_{s}\left(r_{2}-1\right)\right)}\left(\sum_{j=1}^{N} Z_{i, j}^{(1)} C_{*}\left(y_{j}, t\right)\right)+\frac{1}{F r} \\
& -\frac{1+C_{s}\left(r_{1}-1\right)}{\operatorname{Re}{ }^{*} \operatorname{Ca}\left(1+C_{s}\left(r_{2}-1\right)\right)}\left(\sum_{j=1}^{N} Z_{i, j}^{(2)} C_{i}\left(y_{j}, t\right)\right)
\end{aligned}
$$

Thus, (31)-(34) is a reduced system of ordinary differential equations in time, that is, represented as for $i=$ $1,2,3 \ldots, N$.

$$
U_{t}=R\left(u_{1}, C_{*}, C_{i}, u^{*}\right)
$$

The system is solved by the following strong stability b-spline RK43 scheme. $^{52,53}$

$$
U_{1}=U_{0}+\frac{\Delta t}{2} * R\left(u_{10}, C_{* 0}, C_{i 0}, u_{0}^{*}\right)
$$

\section{RESULTS AND DISCUSSION}

According to the time-dependent pressure gradient, the unstable flow of two immiscible micropolar and Newtonian fluids with mutual momentum transfer and viscosity jump across the interface is considered. The traditional no-slip and hyper-stick conditions on the boundaries of the channel were presumed to describe the solution. Single mean flow problems with moving interface have been addressed, and the interface tracking profiles have been acquired by MCB-DQM. The ratio of viscous drag forces and surface tension forces acting at an interface between two immiscible fluids is known as the capillary number $\left(\boldsymbol{C} \boldsymbol{a}=\left(\boldsymbol{\mu}^{*} \boldsymbol{U}_{\boldsymbol{0}}\right) / \boldsymbol{\sigma}\right)$. The current numerical technique is validated by comparing the approximated numerical result for a single non-micropolar Newtonian fluid $\left(\boldsymbol{r}_{1}=1, \boldsymbol{r}_{2}=1\right.$, $\boldsymbol{n}_{1}=0$ ) with the exact solution. Note that if $\boldsymbol{r}_{1}=\boldsymbol{r}_{2}=$ 1 , then the interfacial force $\boldsymbol{\sigma}=0$ and hence $(1 / \boldsymbol{C a})=$ $0,(1 / \boldsymbol{F r})=0$. Figures 2,3 shows the comparative analysis of calculated and exact solutions for velocity profiles at different times and discretized nodes. It is noticed that the applied numerical technique results achieve very good corroboration with the exact solution. The root means square error between analytical and calculated results is very less and also decreases with time and discretized node value. 


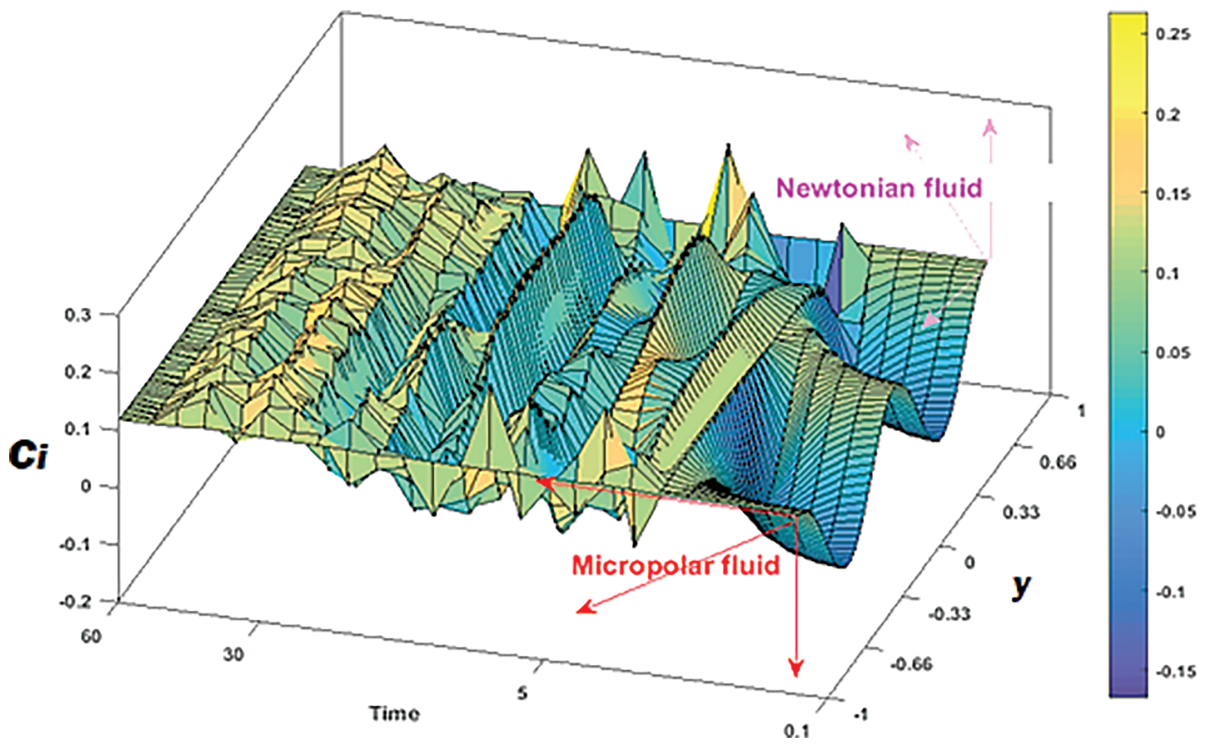

Fig. 12. Interface tracking with varying time when the flow is carried by periodic pressure gradient $\boldsymbol{G e}=\mathbf{1 0} * \operatorname{Sin}(\boldsymbol{w t})$.

Figures 4-14 shows the evolution of the interface between two liquids with varying time, amplitude, and wavenumber under the applied constant, periodic, and decaying pressure gradient. It is evident from Figures 4-9 that, in the case of applied constant pressure gradients, initially, the vertical elongation of the interface is large, and then the shape of the interface evolves with time; hence the undulating sequence occurs faster for a more considerable time, and the interface becomes stable for high time. Note that the top fluid is even less viscous than the bottom fluid. It is also noticed that the qualitative characteristics of this flow shall be maintained, and as expected, the interface starts to shift vertically if the amplitude and wavenumber are enhanced (see Figs. 10 and 11). The two liquids do not penetrate each other, in the same way. The evolution of the interface profile for decaying pressure gradient cases is qualitatively similar, although greater elapse of time is required for it to become stable, and the crest of the waves is smaller compared to the constant pressure case see Figure 12 . It is also worth mentioning that the evolution of the interface profile for the periodic pressure gradient case is qualitatively similar to other cases; however, it takes more time to stabilize, and the crest of the waves is large compared to the constant and decaying pressure case (see Fig. 13). It is also noticed from Figure 14 that the interface becomes stable quickly with time as the micropolar parameter is reduced i.e., weaker micropolar fluids encourage faster stabilization of the interface.

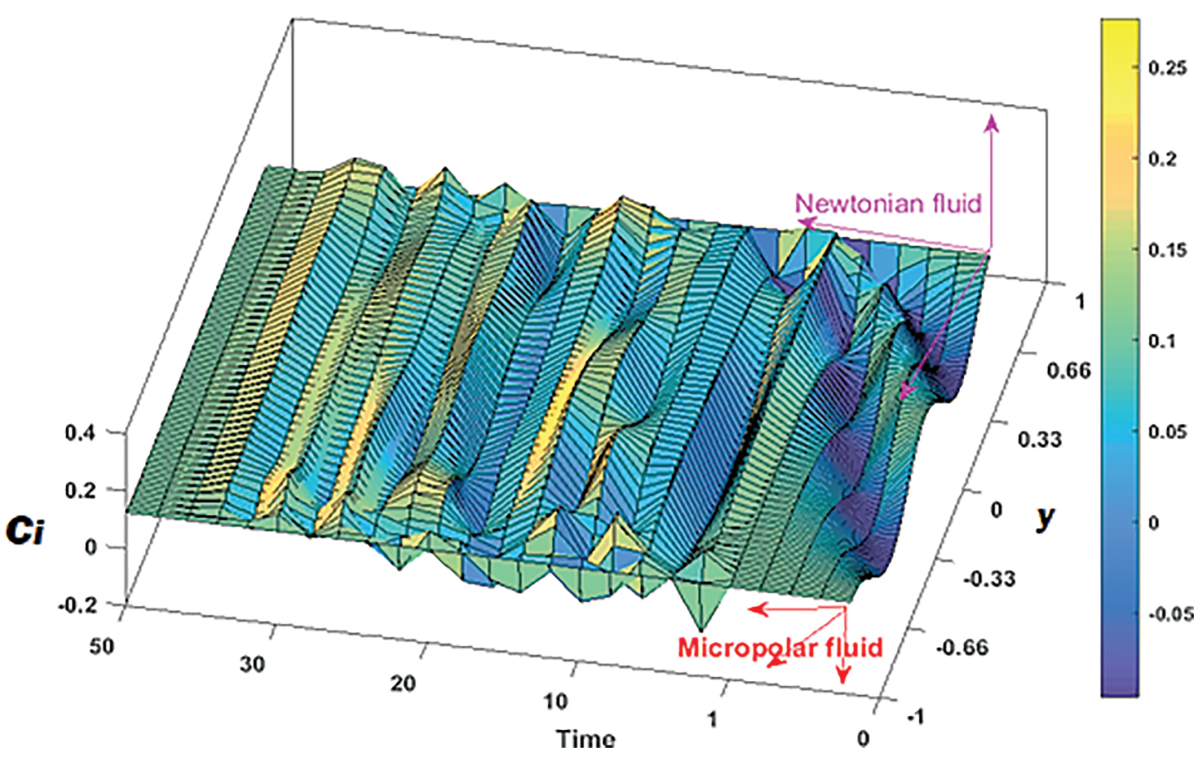

Fig. 13. Interface tracking with varying time when the flow is carried by periodic pressure gradient. $G e=10 * e^{-\lambda t}$. 


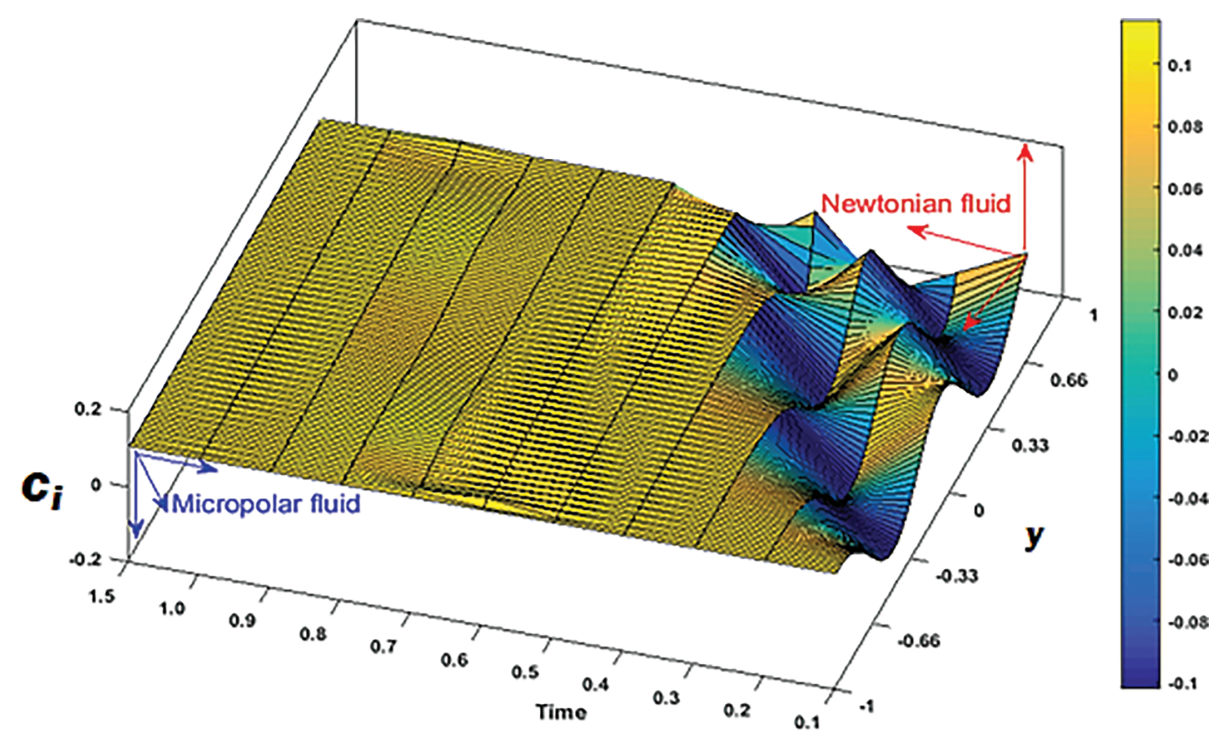

Fig. 14. Interface tracking with varying time when $G e=10, R e=2, \boldsymbol{r}_{1}=0.5, \boldsymbol{r}_{2}=0.5, \boldsymbol{C}_{s}=0.5, \boldsymbol{C a}=0.5, \boldsymbol{F r}=\mathbf{2}$, and micropolar parameter curtailed as $\boldsymbol{n}_{\mathbf{1}}=\mathbf{0 . 0 0 5}$

\section{CONCLUSIONS}

The unsteady flow of two immiscible (micropolar and Newtonian) fluids through a horizontal channel under a moving interface has been analyzed numerically using the method of modified Cubic B-Spline Differential Quadrature. The effects of key parameters such as time, amplitudes, wave number, and micropolar parameter on interface evolution have been addressed with three different applied pressure gradients. The core findings of the present article can be summarized in the following points: - Initially, the vertical stretching of the interface is enormous, and afterward, its shape advances with time; subsequently, the undulating succession occurs quicker, and the interface becomes steady for a more extensive time when a constant pressure gradient is applied.

- The development of the interface profile for decaying pressure gradient cases is subjectively comparative; however, it requires more time to achieve the steady-state, and the peak of the waves resembles those for the constant pressure gradient case.

- The interface profile for periodic pressure gradient cases is qualitatively similar to the other cases; however, it takes more time to stabilize, and the crest of the waves is large compared to the constant and decaying pressure case.

- The interface becomes stable quickly with time as the micropolar parameter is curtailed for the case where the flow is induced by a constant pressure gradient.

The present computations have shown that MCB-DQM is a robust numerical approach for simulating complex interfacial immiscible flows of Newtonian and nonNewtonian fluids. The present study may, however, be extended to consider nanofluids and porous media ${ }^{56}$ and also more complex geometries ${ }^{57}$ e.g., micro-channels, and efforts in these directions are currently underway.

\section{NOMENCLATURE}

$\rho_{2}$ The density co-efficient $\mathrm{kg} / \mathrm{m}^{3}$

$u$ The velocity of the fluid $\mathrm{m} / \mathrm{s}$

$\omega$ Microrotations of lower region fluids $\mathrm{s}^{-1}$

$\gamma_{1},{ }^{\mu}$ and $\delta$ The viscosity coefficients $\mathrm{kg} \cdot \mathrm{m}^{-1} \cdot \mathrm{s}^{-1}$

$u_{1}, u_{2}$ Velocities of lower and upper region fluids $\mathrm{m} / \mathrm{s}$

$\rho_{1}, \rho_{2}$ The density of lower and upper region fluids $\mathrm{kg} / \mathrm{m}^{3}$

$\mu_{1}, \mu_{2}$ The viscosity of lower and upper region fluids $\mathrm{kg} \cdot \mathrm{m}^{-1} \cdot \mathrm{s}^{-1}$

$u^{*} \quad$ The mean flow velocity field in the channel $\mathrm{m} / \mathrm{s}$

$\rho^{*} \quad$ The average density of the flow in the channel $\mathrm{kg} / \mathrm{m}^{3}$

$\mu^{*} \quad$ Average viscosity of flow in the channel $\mathrm{kg}$. $\mathrm{m}^{-1} \cdot \mathrm{s}^{-1}$

$g$ The gravitational acceleration $\mathrm{m} / \mathrm{s}^{2}$

$\sigma$ The interfacial tension between two liquid phases $m N / m$

$l_{1}$ The height of the interface at any point $m$

$y_{m}$ The average depth of the interface $m$

$A_{0} \quad$ The amplitude $m$

$p \quad$ The fluid pressure at any point $\mathrm{Pa}$

$\xi \quad$ The body couple per unit mass

$C_{s}$ The volume fraction function

$C_{i}$ The interface tracking function

$r_{1} \quad$ Ratio of viscosities

$r_{2}$ Ratio of densities

Re Reynolds number

$B_{0}$ wavenumber 
Fr Froude number

$n_{1} \quad$ The microrotation parameter

$f \quad$ The body force per unit mass

$s$ The mean free surface curvature

$\tau$ The gyration co-efficient

$\lambda_{1}, \lambda_{2}, \lambda_{3}$ The gyro-viscosity coefficients.

\section{Ethical Compliance}

This article does not contain any studies involving animals or humans performed by any of the authors.

\section{Conflicts of Interest}

The authors declare that they have no conflicts of interest.

Acknowledgments: The authors of this paper would like to pay sincere thanks to all the reviewers for giving valuable feedback to improve the manuscript. The authors declare that there is no funding source or any grant associated with this research article.

\section{References and Notes}

1. A. C. Eringen, Mechanics of Generalized Continua. IUTAM Symposium (1968), pp.18-35.

2. J. Peddieson, Int. J. Eng. Sci. 10, 23 (1972)

3. O. A. Bég, V. Ramachandra Prasad, B. Vasu, N. Bhaskar Reddy, Q. Li, and R. Bhargava, Int. J. Heat Mass Transf. 54, 9 (2011).

4. C. Kang and A. Eringen, Bull. Math. Biol. 38, 135 (1976).

5. M. Devakar and T. K. V. Iyengar, Eur. Phys. J. Plus 128, 1 (2013).

6. K. S. Mekheimer and M. A. E. Kot, Acta Mech. Sin. Xuebao 24, 637 (2008).

7. D. Srinivasacharya and M. Shiferaw, Arab. J. Sci. Eng. 39, 5085 (2014).

8. L. Wang, Y. Jian, and F. Li, Eur. Phys. J. Plus 131, 1 (2016)

9. F. Selimefendigil, H. F. Öztop, and A. J. Chamkha, Int. J. Numer. Methods Heat Fluid Flow 30, 1755 (2020).

10. A. S. Dogonchi, T. Tayebi, N. Karimi, A. J. Chamkha, and H. Alhumade, J. Taiwan Inst. Chem. Eng. 124, 162 (2021).

11. Y. Menni, A. Azzi, and A. Chamkha, J. Therm. Anal. Calorim. 135, 1951 (2019).

12. J. Raza, F. Mebarek-Oudina, and A. J. Chamkha, Multidiscip. Model. Mater. Struct. 5, 737 (2019).

13. M. Veera Krishna and A. J. Chamkha, Results Phys. 15, 102652 (2019).

14. Y. Menni, A. J. Chamkha, N. Massarotti, H. Ameur, N. Kaid, and M. Bensafi, Int. J. Numer. Methods Heat Fluid Flow 30, 4349 (2020).

15. N. S. Shashikumar, B. J. Gireesha, B. Mahanthesh, P. B. C, and A. J. Chamkha, Int. J. Numer. Methods Heat Fluid Flow 29, 3638 (2019).

16. B. Kumar, G. S. Seth, R. Nandkeolyar, and A. J. Chamkha, Int. J. Therm. Sci. 146, 106101 (2019).

17. Y. Menni, A. Azzi, and A. Chamkha, Int. J. Numer. Methods Heat Fluid Flow 29, 1815 (2019).

18. A. J. Chamkha, I. V. Miroshnichenko, and M. A. Sheremet, J. Therm. Sci. Eng. Appl. 9, 041004 (2017).

19. S. Parvin and A. J. Chamkha, Int. Commun. Heat Mass Transf. 54, 8 (2014).

20. Y. Menni, A. J. Chamkha, and A. Azzi, J. Appl. Comput. Mech. 6, 741 (2020)

21. A. J. Chamkha, Numer. Heat Transf. Part A Appl. 32, 653 (1997).

22. A. J. Chamkha, Int. J. Heat Mass Transf. 45, 2509 (2002).

23. A. J. Chamkha, Int. J. Heat Fluid Flow 21, 740 (2000).
24. M. Ghalambaz, A. Doostani, E. Izadpanahi, and A. J. Chamkha, J. Therm. Anal. Calorim. 139, 2321 (2020).

25. A. J. Chamkha, M. A. Mansour, A. M. Rashad, H. Kargarsharifabad, and T. Armaghani, J. Thermophys. Heat Transf. 34, 836 (2020).

26. S. Parvin, R. Nasrin, M. A. Alim, N. F. Hossain, and A. J. Chamkha, Int. J. Heat Mass Transf. 55, 5268 (2012).

27. P. S. Reddy, P. Sreedevi, and A. J. Chamkha, Powder Technol. 307, 46 (2017).

28. A. J. Chamkha, Appl. Math. Model. 21, 603 (1997).

29. R. B. Bird, Applied Mechanics Reviews 55, R1 (2002).

30. J. C. Umavathi, A. J. Chamkha, M. H. Manjula, and A. Al-Mudhaf, Can. J. Phys. 83, 705 (2005).

31. J. C. Umavathi, J. Prathap Kumar, and A. J. Chamkha, Turkish J. Eng. Environ. Sci. 33, 221 (2009).

32. J. P. Kumar, J. C. Umavathi, A. J. Chamkha, and I. Pop, Appl. Math Model. 34, 1175 (2010).

33. J. Srinivas and J. V. R. Murthy, J. Appl. Fluid Mech. 9, 501 (2016)

34. J. Srinivas, J. V. Ramana Murthy, and K. S. Sai, Comput. Therm. Sci. 7 (2015), DOI: 10.1615/ComputThermalScien.2015012175.

35. J. Srinivas and J. V. Ramana Murthy, J. Appl. Mech. Tech. Phys 57, 997 (2016).

36. A. Borrelli, G. Giantesio, and M. C. Patria, J. Fluids Eng. Trans ASME 139 (2017), DOI: 10.1115/1.4036670.

37. W. Tauber, S. O. Unverdi, and G. Tryggvason, Phys. Fluids 14, 2871 (2002).

38. C. S. Yih, J. Fluid Mech. 27, 337 (1967).

39. R. B. DeBar, Fundamentals of the KRAKEN code. [Eulerian hydrodynamics code for compressible nonviscous flow of several fluids in two-dimensional (axially symmetric) region] (1974), (No. UCID17366). California Univ., Livermore (USA). Lawrence Livermore Lab.

40. D. L. Youngs, Time-dependent multi-material flow with large fluid distortion (1982).

41. J. Li, Comptes Rendus-Academie des Sciences, Serie II: Mecanique, Physique, Chimie, Astronomie 320, 391 (1995).

42. J. Li and Y. Renardy, SIAM Rev. 42, 417 (2000).

43. V. R. Gopala and B. G. M. van Wachem, Chem. Eng. J. 141, 204 (2008).

44. G. Tryggvason, B. Bunner, A. Esmaeeli, D. Juric, N. Al-Rawahi, W. Tauber, J. Han, S. Nas, and Y.-J. Jan, J. Comput. Phys. 169, 708 (2001).

45. A. Riaz and H. A. Tchelepi, Phys. Fluids 18, 014104 (2006).

46. K. Vajravelu, P. V. Arunachalam, and S. Sreenadh, J. Math. Anal. Appl. 196, 1105 (1995).

47. J. C. Umavathi, A. J. Chamkha, A. Mateen, and A. Al-Mudhaf, Heat Mass Transf. und Stoffuebertragung 42, 81 (2005).

48. M. Devakar and A. Raje, Eur. Phys. J. Plus 133, 180 (2018)

49. R. Bellman, B. G. Kashef, and J. Casti, J. Comput. Phys. 10, 40 (1972).

50. J. R. Quan and C. T. Chang, Comput. Chem. Eng. 13, 1017 (1989).

51. C. Shu and B. E. Richards, Int. J. Numer. Methods Fluids 15, 791 (1992).

52. R. Katta, R. K. Chandrawat, and V. Joshi, A Numerical Study of the unsteady flow of two immiscible micropolar and Newtonian fluids through a horizontal channel using DQM with B-Spline basis function (2020), DOI: 10.1088/1742-6596/1531/1/012090.

53. K. Ramesh and V. Joshi, Int. J. Comput. Methods Eng. Sci. Mech 20, 1 (2019).

54. H. Tang and L. C. Wrobel, Int. J. Eng. Sci. 43, 1234 (2005).

55. R. K. Chandrawat, V. Joshi, and O. A. Beg, Int. J. Comput. Methods Eng. Sci. Mech. 10 (2019), DOI: 10.1080/15502287.2018.1520322.

56. Y. Menni, A. J. Chamkha, and A. Azzi, Spec. Top. Rev. Porous Media 10 (2019), DOI: 10.1615/SpecialTopicsRevPorousMedia.2018027168.

57. Y. Menni, A. J. Chamkha, and A. Azzi, J. Nanofluids 8, 893 (2019). 